Article

\title{
Gradient Structures Associated with a Polynomial Differential Equation
}

\author{
Savin Treanţă (1) \\ Department of Applied Mathematics, University Politehnica of Bucharest, 060042 Bucharest, Romania; \\ savin.treanta@upb.ro
}

Received: 19 March 2020; Accepted: 31 March 2020; Published: 4 April 2020

check for updates

\begin{abstract}
In this paper, by using the characteristic system method, the kernel of a polynomial differential equation involving a derivation in $R^{n}$ is described by solving the Cauchy Problem for the corresponding first order system of PDEs. Moreover, the kernel representation has a special significance on the space of solutions to the corresponding system of PDEs. As very important applications, it has been established that the mathematical framework developed in this work can be used for the study of some second-order PDEs involving a finite set of derivations.
\end{abstract}

Keywords: scalar derivation; Lie algebra; gradient system; polynomial differential equation; flow; kernel

MSC: 34A26; 15A03; 35C99

\section{Introduction}

Throughout time, gradient-type representations for some solutions, gradient systems in a Lie algebra and the algebraic representation of gradient systems, have been investigated, with remarkable results, by Vârsan [1]. Moreover, stochastic partial differential equations (SPDEs) of Hamilton-Jacobi type including non $F_{T}$-adapted solutions have been studied in Ijacu and Vârsan [2]. By using the commuting property of the drift and diffusion vector fields with respect to the usual Lie bracket, a representation for a classical solution of some nonlinear SPDEs driven by Fisk-Stratonovich stochastic integral was constructed by Iftimie et al. [3]. Furthermore, sufficient conditions for linear subspaces of smooth vector fields in order to be written as a kernel of some linear first order partial differential equations are have been formulated and proved in Parveen and Akram [4]. Further, Treanţă and Vârsan [5] proved that solutions associated with an extended affine control system can be obtained as a limit process using solutions for a parameterized affine control system and weak small controls. Recently, Treanţă [6] studied affine control systems with jumps for which the ideal generated by the drift vector field can be imbedded as a kernel of a linear first-order partial differential equation. Mainly, these references motivate the present study. For other different but connected viewpoints regarding this subject, the reader is directed to Friedman [7], Sussmann [8], Crandall and Souganidis [9], Sontag [10], Bressan and Shen [11], Nonlaopon [12], Saira et al. [13] and Treanţă [14-16].

In this paper, taking into account the results included in the quite recently work Treanţă [17] (the kernel of a polynomial of scalar derivations is described by solving Cauchy Problems for the corresponding system of ODEs; also, a gradient representation for the associated Cauchy Problem solution is derived), we investigate the kernel of a polynomial differential equation involving a derivation in $R^{n}$ by solving the Cauchy Problem for the corresponding first order system of PDEs. Furthermore, we extend a solution 
by considering Radon measures and their bounded variation functions, or Wiener and Levy processes. Moreover, it is established that the kernel representation has a special significance on the space of solutions to the corresponding system of PDEs.

This paper is organized as follows. In Section 2, in order to delineate certain steps in the solving algorithm proposed for the main result (Theorem 1), some preliminary results are formulated. More precisely, two crucial lemmas for the present paper are mentioned. Further, we establish two important remarks. A gradient structure for the associated Cauchy Problem solution is provided by Remark 1. The final part of this section, including Remark 2, extends a solution considering Radon measures and their bounded variation functions, or using Wiener (or Levy) processes. The aim of Section 3 is to provide a characterization for the kernel of a polynomial differential equation involving a derivation in $R^{n}$. Specifically, through the use of the characteristic system method and some results formulated in Section 2, the associated Cauchy Problem solution is derived (see Theorem 1). Moreover, this solution has a special significance on the space of solutions to the corresponding first order system of PDEs. Finally, Section 4 concludes the paper.

\section{Preliminary Results}

In this section, taking into account a very recent work (see Treanţă [17]), some auxiliary results are formulated.

Let $0 \in I \subseteq R$ be an open interval. Consider a polynomial of the scalar derivation $\frac{d}{d t}$,

$$
P_{m}\left(t ; \frac{d}{d t}\right)=a_{1}(t)+a_{2}(t)\left(\frac{d}{d t}\right)+\cdots+a_{m}(t)\left(\frac{d}{d t}\right)^{m-1}-\left(\frac{d}{d t}\right)^{m},
$$

where $m \geq 1, a_{j} \in L_{\infty}(I), j \in\{1,2, \ldots, m\}$. Define

$$
H_{\infty}^{m}(I)=\left\{h \in C^{m-1}(I):\left(\frac{d}{d t}\right)^{m}(h) \in L_{\infty}(I)\right\}
$$

and consider $\operatorname{Ker}\left(P_{m}\right) \subseteq H_{\infty}^{m}(I)$, where

$$
\operatorname{Ker}\left(P_{m}\right)=\left\{h \in H_{\infty}^{m}(I): P_{m}\left(t ; \frac{d}{d t}\right)(h)(t)=0 \text {, a.e. } t \in I\right\} .
$$

The procedure of characteristic systems (see Friedman [7], Vârsan [1]) allows us to describe $\operatorname{Ker}\left(P_{m}\right)$ by solving Cauchy Problems for the corresponding system of ODEs using a vector variable

$$
y=\operatorname{col}\left(y_{1}, y_{2}, \ldots, y_{m}\right), \frac{d y}{d t}=A y+\sum_{i=1}^{m} a_{i}(t) B_{i} y, y(0)=y_{0} \in R^{m}
$$

Here, the $(m \times m)$ constant matrices $A$ and $B_{i}, i=\overline{1, m}$, are defined by

$$
A=\left[\begin{array}{llll}
0 & e_{1} & \cdots & e_{m-1}
\end{array}\right], B_{1}=\left[\begin{array}{llll}
e_{1} & 0 & \cdots & 0
\end{array}\right], \cdots, B_{m}=\left[\begin{array}{llll}
0 & 0 & \cdots & e_{m}
\end{array}\right],
$$

where $\left\{e_{1}, \ldots, e_{m}\right\}$ is the canonical basis and $0 \in R^{m}$ is the origin. By definition

$$
\left[B_{i}, B_{j}\right]:=B_{j} B_{i}-B_{i} B_{j}, i, j \in\{1,2, \ldots, m\}, \quad \text { (Lie bracket), }
$$


and making a direct computation, we get

$$
O=\left[B_{i}, B_{j}\right], i, j \in\{1,2, \ldots, m\},
$$

with $O$-null matrix, and

$$
A^{m}=O, \quad(A \text { is a nilpotent matrix }) .
$$

The Cauchy Problem solution for (4) is represented by

$$
y\left(t ; y_{0}\right)=[\exp A t] \hat{y}\left(t ; y_{0}\right), t \in I,
$$

where $\left\{\hat{y}\left(t ; y_{0}\right): t \in I\right\}$ fulfils the following linear system (initial value problem)

$$
\frac{d y}{d t}=\sum_{i=1}^{m} a_{i}(t) A_{i}(t) y, t \in I, y(0)=y_{0} \in R^{m}
$$

Write the $(m \times m)$ matrices

$$
A_{i}(t):=[\exp (-t A)] B_{i}[\exp t A], i \in\{1,2, \ldots, m\},
$$

as follows

$$
A_{i}(t)=\left[\exp t \operatorname{ad}_{A}\right]\left(B_{i}\right), i \in\{1,2, \ldots, m\}, t \in I,
$$

where the linear mapping $\operatorname{ad}_{A}: M_{m \times m} \rightarrow M_{m \times m}$ is given by $\operatorname{ad}_{A}(B):=B A-A B$ (see $[A, B]$ ). In addition, using (7), (8) and (12), we get

$$
A_{i}(t)=B_{i}+\frac{t}{1 !} \operatorname{ad}_{A}\left(B_{i}\right)+\cdots+\frac{t^{m-1}}{(m-1) !}\left(\operatorname{ad}_{A}\right)^{m-1}\left(B_{i}\right), i \in\{1, \ldots, m\} .
$$

Denote $N=m^{2}$ and define $N$ matrices $\left\{C_{1}, C_{2}, \ldots, C_{N}\right\} \subseteq M_{m \times m}$, as follows

$$
\begin{gathered}
\left\{C_{1}, C_{2}, \ldots, C_{N}\right\}=\left\{a d_{A}^{k}\left(B_{1}\right): k \in\{0,1,2, \ldots, m-1\}\right\} \cup \cdots \cup \\
\left\{a d_{A}^{k}\left(B_{m}\right): k \in\{0,1,2, \ldots, m-1\}\right\} .
\end{gathered}
$$

Furthermore, let $\left\{\alpha_{1}(t), \alpha_{2}(t), \ldots, \alpha_{N}(t): t \in I\right\}$ be given by

$$
\begin{gathered}
\left\{\alpha_{1}(t), \ldots, \alpha_{m}(t)\right\}=a_{1}(t)\left[1, \frac{t}{1 !}, \ldots, \frac{t^{m-1}}{(m-1) !}\right], \\
\vdots \\
\left\{\alpha_{N-m+1}(t), \ldots, \alpha_{N}(t)\right\}=a_{m}(t)\left[1, \frac{t}{1 !}, \ldots, \frac{t^{m-1}}{(m-1) !}\right] .
\end{gathered}
$$

With these notations, we write ODE (10) as follows

$$
\frac{d y}{d t}=\sum_{j=1}^{N} \alpha_{j}(t) Y_{j}(y), t \in I, y(0)=y_{0},
$$

where $Y_{j}(y):=C_{j} y, j \in\{1,2, \ldots, N\}$. 
Lemma 1 ([17]). Consider $\left\{C_{1}, C_{2}, \ldots, C_{N}\right\}$ defined in (14), with $N=m^{2}$. Then $\left\{C_{1}, C_{2}, \ldots, C_{N}\right\}$ is a basis for $M_{m \times m}$ and

$$
\left\{Y_{1}(y)=C_{1} y, Y_{2}(y)=C_{2} y, \ldots, Y_{N}(y)=C_{N} y\right\}
$$

is a system of generators for the Lie algebra $L\left(Y_{1}, \ldots, Y_{N}\right) \subseteq C^{\infty}\left(R^{m} ; R^{m}\right)$ generated by $\left\{Y_{1}, \ldots, Y_{N}\right\}$.

Lemma 2 ([17]). Assume $a_{j}(t) \equiv 0, j \in\{1,2, \ldots, i\}$, for some $1 \leq i \leq m$. Define a subspace $S_{i}=$ span $\left\{e_{1}, \ldots, e_{i}\right\} \subseteq R^{m}$ and its orthogonal complement $S_{i}^{\perp} \subseteq R^{m}$, where $\left\{e_{1}, \ldots, e_{m}\right\} \subseteq R^{m}$ is the canonical basis. Then

$$
\forall y_{0} \in S_{i} \text { is a stationary point for ODE (10). }
$$

In addition, for each $y_{0} \in S_{i}^{\perp}, y_{0} \neq 0$, the following statements are valid:

$$
\begin{gathered}
\operatorname{dim} L\left(Y_{j(i)}, \ldots, Y_{N}\right)\left(y_{0}\right)=\operatorname{dim}\left[\operatorname{span}\left\{Y_{j(i)}\left(y_{0}\right), \ldots, Y_{N}\left(y_{0}\right)\right\}\right]=m-i, \\
\left\{C_{j(i)}, \ldots, C_{N}\right\} \text { is a basis for } M_{m \times m}^{i}:=L\left(C_{j(i)}, \ldots, C_{N}\right), j(i):=m i+1 \\
\hat{y}\left(t, y_{0}\right) \in S_{i}^{\perp}, t \in I, \text { for any solution of } O D E(10) .
\end{gathered}
$$

In the following, we establish two important remarks for the main result associated with this paper.

Remark 1. Any solution of ODE (4) can be represented using

$$
p(t):=\left(t_{1}(t), \ldots, t_{m}(t)\right), t_{j}(t)=\int_{0}^{t} a_{j}(s) d s, j \in\{1,2, \ldots, m\}, t \in I .
$$

In this regard, notice that the matrices $\left\{B_{1}, \ldots, B_{m}\right\} \subseteq M_{m \times m}$ commute (see (6)) and the linear mapping

$$
G(p) \lambda:=\left[\exp t_{1} B_{1}\right] \cdots\left[\exp t_{m} B_{m}\right] \lambda, p=\left(t_{1}, \ldots, t_{m}\right) \in R^{m}, \lambda \in R^{m}
$$

satisfies a gradient system

$$
\partial_{t_{1}} G(p) \lambda=B_{1} G(p) \lambda, \cdots, \partial_{t_{m}} G(p) \lambda=B_{m} G(p) \lambda
$$

In particular, for $p=p(t), t \in I$ (see (20)), we get that any $\left\{y\left(t, y_{0}\right): t \in I\right\}$ satisfying ODE (4) is represented by

$$
y\left(t ; y_{0}\right)=G(p(t)) \hat{y}\left(t ; y_{0}\right), t \in I, y_{0} \in R^{m},
$$

where $\left\{\hat{y}\left(t ; y_{0}\right): t \in I\right\} \subseteq C\left(I, R^{m}\right)$ fulfils

$$
\frac{d y}{d t}=G(-p(t)) A G(p(t)) y, y(0)=y_{0}, t \in I
$$

In addition, $A(t):=G(-p(t)) A G(p(t)), t \in I$, defined in (24), can be written as (see "o" as the symbol for composing functions)

$$
A(t)=\left[\exp t_{1}(t) \operatorname{ad}_{B_{1}}\right] \circ \ldots \circ\left[\exp t_{m}(t) \operatorname{ad}_{B_{m}}\right](A), t \in I,
$$


where the adjoint mapping $\operatorname{ad}_{B}: M_{m \times m} \rightarrow M_{m \times m}$ is defined by

$$
\operatorname{ad}_{B}(C)=[B, C]=C B-B C, \quad(\text { Lie bracket }) .
$$

A direct computation leads us to

$$
\left[\exp t \operatorname{ad}_{B_{m}}\right](A)=A+\frac{t}{1 !} \operatorname{ad}_{B_{m}}(A)+\cdots+\frac{t^{k}}{k !} a d_{B_{m}}^{k}(A)+\cdots,
$$

where $a d_{B_{m}}^{k}(A)=C_{m}:=\left[\begin{array}{lll}0 & 0 & e_{m-1}\end{array}\right]$, for any $k \geq 1$. Rewrite (27) as

$$
\left[\exp t \operatorname{ad}_{B_{m}}\right](A)=A+[\exp t-1] C_{m}, C_{m}=\left[\begin{array}{llll}
0 & \ldots & 0 & e_{m-1}
\end{array}\right] .
$$

Notice that

$$
\operatorname{ad}_{B_{m-1}}\left(C_{m}\right)=C_{m-1}-B_{m}, \quad \operatorname{ad}_{B_{m-1}}\left(C_{m-1}\right)=O,
$$

where $C_{m-1}:=\left[\begin{array}{lllll}0 & \ldots & 0 & e_{m-1} & 0\end{array}\right]$. Using (28) and (29), we obtain

$$
\left[\begin{array}{lll}
\exp & t_{m-1} & \operatorname{ad}_{B_{m-1}}
\end{array}\right]\left(C_{m}\right)=C_{m}+t_{m-1}\left(C_{m-1}-B_{m}\right)
$$

and

$$
\begin{aligned}
& {\left[\begin{array}{lll}
\exp & t_{m-1} & \operatorname{ad}_{B_{m-1}}
\end{array}\right] \circ\left[\begin{array}{lll}
\exp & t_{m} & \operatorname{ad}_{B_{m}}
\end{array}\right](A)} \\
& =\left[\left(\begin{array}{lll}
\exp & t_{m}
\end{array}\right)-1\right]\left[\begin{array}{lll}
\exp t_{m-1} & \operatorname{ad}_{B_{m-1}}
\end{array}\right]\left(C_{m}\right)+\left[\begin{array}{lll}
\exp t_{m-1} & \operatorname{ad}_{B_{m-1}}
\end{array}\right](A) \\
& =\left[\left(\exp t_{m}\right)-1\right]\left[C_{m}+t_{m-1}\left(C_{m-1}-B_{m}\right)\right]+\left[\exp t_{m-1} \operatorname{ad}_{B_{m-1}}\right](A) \text {. }
\end{aligned}
$$

On the other hand, compute

$$
\left[\exp t_{m-1} \operatorname{ad}_{B_{m-1}}\right](A)=A+t_{m-1}\left(C_{m-1}-B_{m}\right)-\frac{t_{m-1}^{2}}{2 !} B_{m-1},
$$

where $C_{m-1}=\left[\begin{array}{lllll}0 & \ldots & 0 & e_{m-1} & 0\end{array}\right]$, and inserting (31) into (30), we get

$$
\begin{gathered}
{\left[\exp t_{m-1} \mathrm{ad}_{B_{m-1}}\right] \circ\left[\exp t_{m} \operatorname{ad}_{B_{m}}\right](A)} \\
=A+\left(\exp t_{m}\right)\left[C_{m}+t_{m-1}\left(C_{m-1}-B_{m}\right)\right]-C_{m}-\frac{t_{m-1}^{2}}{2 !} B_{m-1} .
\end{gathered}
$$

Notice that (see (31))

$$
\left[\exp t_{m-2} \operatorname{ad}_{B_{m-2}}\right](A)=A+t_{m-2}\left(C_{m-2}-B_{m-1}\right),
$$

and applying [exp $\left.t_{m-2} \quad a_{B_{m-2}}\right]$ to (32), we obtain

$$
\begin{gathered}
{\left[\exp t_{m-2} \operatorname{ad}_{B_{m-2}}\right] \circ\left[\exp t_{m-1} \operatorname{ad}_{B_{m-1}}\right] \circ\left[\exp t_{m} \operatorname{ad}_{B_{m}}\right](A)} \\
=A+t_{m-2}\left(C_{m-2}-B_{m-1}\right)+\left(\exp t_{m}\right)\left[C_{m}+t_{m-1}\left(C_{m-1}-B_{m}\right)\right] \\
-C_{m}-\frac{t_{m-1}^{2}}{2 !} B_{m-1}+t_{m-2}\left[\left(\exp t_{m}\right)-1\right] C_{m-2} .
\end{gathered}
$$


This computation shows that

$$
\hat{A}\left(t_{1}, \ldots, t_{m}\right):=\left[\exp t_{1} \operatorname{ad}_{B_{1}}\right] \circ \ldots \circ\left[\exp t_{m} \operatorname{ad}_{B_{m}}\right](A)
$$

and, in particular, $A(t)=\hat{A}\left(t_{1}(t), \ldots, t_{m}(t)\right)$ (see (25)), can be written as

$$
\hat{A}\left(t_{1}, \ldots, t_{m}\right)=A+\sum_{k=1}^{m} \alpha_{k}\left(t_{1}, \ldots, t_{m}\right) B_{k}+\sum_{k=1}^{m} \beta_{k}\left(t_{1}, \ldots, t_{m}\right) C_{k}
$$

for $\alpha_{k}, \beta_{k} \in C^{\omega}\left(R^{m}\right)$.

Remark 2. Any solution of ODE (4) can be represented as in (23) (see Remark 1) and it allows us to extend a solution considering Radon measures and their bounded variation functions

$$
p(t):=\left(t_{1}(t), \ldots, t_{m}(t)\right), t_{j}(t)=\mu_{j}(t)=\int_{0}^{t} d \mu_{j}(s), j \in\{1,2, \ldots, m\},
$$

with $t \in[0, T] \subseteq I$, replacing $p \in C\left([0, T] ; R^{m}\right)$ defined in (20).

In addition, any solution of ODE (4) can be represented as in (23) using Wiener, $W(t, w):[0, T] \times \Omega \rightarrow$ $R^{m}$, or Levy processes, $p(t, w):[0, T] \times \Omega \rightarrow R^{m}$, replacing $p \in C\left([0, T] ; R^{m}\right)$ in $(20)$. If it is the case, then any solution of ODE (4) and, as a consequence, $\operatorname{Ker}\left(P_{m}\right)$ defined in (3), changes accordingly. We get (see (23))

$$
\begin{aligned}
& y\left(t, w ; y_{0}\right)=G(W(t, w)) \hat{y}\left(t, w ; y_{0}\right), t \in[0, T], \\
& y\left(t, w ; y_{0}\right)=G(p(t, w)) \hat{y}\left(t, w ; y_{0}\right), t \in[0, T],
\end{aligned}
$$

where $\left\{\hat{y}\left(t, w ; y_{0}\right): t \in[0, T], w \in \Omega\right\}$ is a continuous process satisfying a system of ODEs

$$
\frac{d y}{d t}=G(-\hat{p}(t, w)) A G(\hat{p}(t, w)) y, y(0)=y_{0}, t \in[0, T]
$$

with $\hat{p}(t, w)=W(t, w), t \in[0, T], w \in \Omega$, or $\hat{p}(t, w)=p(t, w), t \in[0, T], w \in \Omega$.

\section{Main Results}

This section contains the main result associated with the present paper. In order to formulate and prove it, we start with the following mathematical tools and hypotheses.

Let $D \subseteq R^{n}$ be an open and convex set. Consider a derivation mapping $\vec{X}: C^{\infty}(D) \rightarrow C^{m}(D)$ given by $\vec{X}(\varphi)(x):=\sum_{i=1}^{n} X_{i}(x) \partial_{i} \varphi(x)$, where $X_{i} \in C^{m}(D), 1 \leq i \leq n, \varphi \in C^{\infty}(D)$. Associate a polynomial differential equation

$$
P_{m}(x ; \vec{X})(\varphi)(x):=f(x)+\sum_{j=1}^{m} a_{j}(x) y_{j}(x)-y_{m+1}(x)=0,
$$

where $f, a_{j} \in C^{1}(D), x \in \hat{B}:=B\left(x_{0}, \rho\right) \subseteq D, y_{1}=\varphi, \vec{X}\left(y_{j}\right)=y_{j+1}$, and $\left(X_{1}\left(x_{0}\right), \ldots, X_{n}\left(x_{0}\right)\right) \neq 0_{R^{n}}$. Assume that

$$
X_{n}(x) \neq 0, \forall x \in B\left(x_{0}, \rho\right) \subseteq D,
$$


where $X=\left(X_{1}, \ldots, X_{n}\right)$. Define a restricted kernel of $P_{m}$ to $\hat{B}$ by

$$
\left.\operatorname{Ker} P_{m}\right|_{\hat{B}}:=\left\{\varphi \in C^{m}(\hat{B} \subseteq D): P_{m}(x ; \vec{X})(\varphi)(x)=0, x \in \hat{B}\right\} .
$$

A procedure for describing $\left\{\left.\operatorname{Ker} P_{m}\right|_{\hat{B}}\right\}$ is to associate a first order system of PDEs involving a vector state variable $y=\left(y_{1}, y_{2}, \ldots, y_{m}\right), y_{i}(x):=(\vec{X})^{(i-1)}(\varphi)(x), 1 \leq i \leq m$. More precisely, consider the following first order system of PDEs

$$
\begin{gathered}
\vec{X}\left(y_{1}\right)(x)=y_{2}(x), \ldots, \vec{X}\left(y_{m-1}\right)(x)=y_{m}(x) \\
\vec{X}\left(y_{m}\right)(x)=f(x)+\sum_{i=1}^{m} a_{i}(x) y_{i}(x) .
\end{gathered}
$$

A Cauchy Problem (CP) for (42) is defined considering a hyperplane $H:=\left\{\left(\hat{x}, x_{0 n}\right) \in R^{n}: \hat{x} \in R^{n-1}\right\}$ (see $\left.x_{0}:=\left(\hat{x}_{0}, x_{0 n}\right)\right)$ and some fixed Cauchy conditions on $H \cap D$

$$
y_{1}\left(\hat{x}, x_{0 n}\right)=y_{1}^{0}(\hat{x}), \ldots, y_{m}\left(\hat{x}, x_{0 n}\right)=y_{m}^{0}(\hat{x}), \hat{x} \in B\left(\hat{x}_{0}, 2 \rho\right) \subseteq R^{n-1},
$$

where $y_{i+1}^{0} \in C^{m-i}\left(B\left(\hat{x}_{0}, 2 \rho\right)\right)$ satisfies $y_{i+1}^{0}=(\vec{X})^{(i)}\left(y_{1}^{0}\right), 1 \leq i \leq m-1$, and $\left\{\left(\hat{x}, x_{0 n}\right): \hat{x} \in B\left(\hat{x}_{0}, 2 \rho\right) \subseteq R^{n-1}\right\} \subseteq H \cap D$.

A solution for (CP) (see (42) and (43)) is found by solving the corresponding characteristic system

$$
\begin{gathered}
\frac{d \hat{y}_{1}}{d \sigma}(\sigma, \lambda)=\hat{y}_{2}(\sigma, \lambda), \ldots, \frac{d \hat{y}_{m-1}}{d \sigma}(\sigma, \lambda)=\hat{y}_{m}(\sigma, \lambda), \\
\frac{d \hat{y}_{m}}{d \sigma}(\sigma, \lambda)=\hat{f}(\sigma, \lambda)+\sum_{i=1}^{m} \hat{a}_{i}(\sigma, \lambda) \hat{y}_{i}(\sigma, \lambda), \sigma \in[-\alpha, \alpha],
\end{gathered}
$$

with Cauchy conditions $\hat{y}_{i}(0, \lambda)=y_{i}^{0}(\lambda), \lambda \in B\left(\hat{x}_{0}, 2 \rho\right) \subseteq R^{n-1}, 1 \leq i \leq m$. Here, the smooth functions $y_{i}^{0}$ are fixed in (43) and $\hat{f}, \hat{a}_{i}$ are given by

$$
\hat{f}(\sigma, \lambda)=f(x(\sigma, \lambda)), \hat{a}_{i}(\sigma, \lambda)=a_{i}(x(\sigma, \lambda)), 1 \leq i \leq m,
$$

for $\sigma \in[-\alpha, \alpha], \lambda \in B\left(\hat{x}_{0}, 2 \rho\right) \subseteq R^{n-1}$, where the local flow $\{x(\sigma, \lambda)\}$ satisfies the following system of ODEs

$$
\frac{d x}{d \sigma}(\sigma, \lambda)=X(x(\sigma, \lambda)), x(0, \lambda)=\left(\lambda, x_{0 n}\right), \lambda \in B\left(\hat{x}_{0}, 2 \rho\right) \subseteq R^{n-1} .
$$

The analysis of ODEs (44) relies on the results derived in Section 2 (see Lemmas 1 and 2 and Remarks 1 and 2$)$, for each $\lambda \in B\left(\hat{x}_{0}, 2 \rho\right)$. This will lead us to the corresponding results for (44) which can be transfered to (42) and (43) provided the local flow $\{x(\sigma, \lambda)\}$ is written as follows

$$
x(\sigma, \lambda)=G(\sigma)\left[\lambda, x_{0 n}\right], \sigma \in[-\alpha, \alpha], \lambda \in B\left(\hat{x}_{0}, 2 \rho\right) \subseteq R^{n-1},
$$

where $\left\{G(\sigma)[z] \in B\left(x_{0}, 3 \rho\right) \subseteq D: \sigma \in[-2 \alpha, 2 \alpha], z \in B\left(x_{0}, 2 \rho\right)\right\}$ is the local flow generated by $X \in C^{m}\left(D \subseteq R^{n} ; R^{n}\right)$, with initial condition $G(0)[z]=z$. In addition, the following equation must be solved

$$
x(\sigma, \lambda)=x \in B\left(x_{0}, \rho\right) \subseteq D, x_{0}:=\left(\hat{x}_{0}, x_{0 n}\right),
$$


for $\lambda=\left(\lambda_{1}, \ldots, \lambda_{m-1}\right) \in B\left(\hat{x}_{0}, 2 \rho\right) \subseteq R^{n-1}$ and $\sigma \in[-\alpha, \alpha]$. The unique solution $\lambda=\hat{\psi}(x)$ and $\sigma=\hat{\sigma}(x)$ of (48) satisfies

$$
\hat{\psi}\left(\hat{x}, x_{0 n}\right)=\hat{x}, \hat{\sigma}\left(\hat{x}, x_{0 n}\right)=0
$$

for $\hat{x} \in B\left(\hat{x}_{0}, 2 \rho\right) \subseteq R^{n-1}$.

For each $x \in \operatorname{Int} B\left(x_{0}, \rho\right)$, let $\left\{G(\sigma)[x] \in B\left(x_{0}, \rho\right): \sigma \in[-\varepsilon, \varepsilon]\right\}$ be the corresponding solution of (46) and notice that (see (47) and (48))

$$
G(\sigma)[x]=G(\sigma) \circ G(\hat{\sigma}(x))\left[\hat{\psi}(x), x_{0 n}\right]=G(\sigma+\hat{\sigma}(x))\left[\hat{\psi}(x), x_{0 n}\right],
$$

for any $\sigma \in[-\varepsilon, \varepsilon]$. Using (50) and the unique solution of (48), we get

$$
\hat{\sigma}(G(\sigma)[x])=\sigma+\hat{\sigma}(x) \in[-\alpha, \alpha], \hat{\psi}(G(\sigma)[x])=\hat{\psi}(x) \in B\left(\hat{x}_{0}, 2 \rho\right),
$$

for any $\sigma \in[0, \varepsilon]$ (or $\sigma \in[-\varepsilon, 0])$.

Define a solution for (CP) (see (42) and (43)) by

$$
y_{i}(x)=\hat{y}_{i}(\hat{\sigma}(x) ; \hat{\psi}(x)), i \in\{1, \ldots, m\}, x \in B\left(x_{0}, \rho\right) \subseteq D,
$$

where $\hat{y}_{i}(\sigma, \lambda), i \in\{1, \ldots, m\}$, fulfil (44) and $\left\{(\hat{\sigma}(x), \hat{\psi}(x)): x \in B\left(x_{0}, \rho\right) \subseteq D\right\}$ verifies (49) and (50).

By a direct inspection, the Cauchy conditions (43) are verified (see (49) and (44)). We get a solution of (42) relying on (51) and

$$
y_{i}(G(\sigma)[x])=\hat{y}_{i}(\sigma+\hat{\sigma}(x) ; \hat{\psi}(x)), i=\overline{1, m}, \sigma \in[0, \varepsilon]
$$

Applying a direct derivation $\left.\frac{d}{d \sigma}\right|_{\sigma=0}$, from (53), we obtain

$$
\begin{gathered}
\left\langle\partial_{x} y_{i}(x), X(x)\right\rangle=y_{i+1}(x), i \in\{1, \ldots, m-1\}, \\
\left\langle\partial_{x} y_{m}(x), X(x)\right\rangle=f(x)+\sum_{i=1}^{m} a_{i}(x) y_{i}(x), x \in \operatorname{Int} B\left(x_{0}, \rho\right) \subseteq D,
\end{gathered}
$$

which stands for the first order system (42).

We are able now to provide the main result of this paper. The above given computation will be stated as follows.

Theorem 1. Let the smooth vector field $X \in C^{m}\left(D \subseteq R^{n} ; R^{n}\right)$ be given such that $X=\left(X_{1}, \ldots, X_{n}\right)$ satisfies $X_{n}\left(x_{0}\right) \neq 0$ for some $x_{0}=\left(\hat{x}_{0}, x_{0 n}\right) \in D$. Consider $(44)$ and its solution

$$
\left\{\hat{y}_{1}(\sigma, \lambda), \ldots, \hat{y}_{m}(\sigma, \lambda): \sigma \in[-\alpha, \alpha], \lambda \in B\left(\hat{x}_{0}, 2 \rho\right) \subseteq R^{n-1}\right\}
$$

Define $y_{i}(x)=\hat{y}_{i}(\hat{\sigma}(x) ; \hat{\psi}(x)), i \in\{1, \ldots, m\}, x \in B\left(x_{0}, \rho\right) \subseteq D$, where $\lambda=\hat{\psi}(x) \in B\left(\hat{x}_{0}, 2 \rho\right) \subseteq R^{n-1}$ and $\hat{\sigma}(x) \in[-\alpha, \alpha]$ is the unique solution of (48) fulfilling (49) and (51). Then $\left\{\left(y_{1}(x), \ldots, y_{m}(x)\right) \in R^{m}: x \in\right.$ $\left.B\left(x_{0}, \rho\right) \subseteq D\right\}$ satisfies the first order system of PDEs given in (42), with Cauchy conditions (43) and $y_{1} \in$ $\left.\operatorname{Ker} P_{m}\right|_{\hat{B}}($ see $(41))$.

Proof. Taking into account the aforementioned computations (see relations (53) and (54)), the proof is immediate and complete. 
Remark 3. Consider a second order PDE of the form

$$
\left\langle\partial_{x}^{2} h(x) X(x), X(x)\right\rangle+\left\langle\partial_{x} h(x),\left[\partial_{x} X(x)\right] X(x)\right\rangle=a_{1}(x) h(x),
$$

where $a_{1} \in C^{1}\left(D \subseteq R^{n}\right)$ and $X \in C^{2}\left(D \subseteq R^{n} ; R^{n}\right)$ is a smooth vector field satisfying $X\left(x_{0}\right) \neq 0_{R^{n}}$ for some $x_{0} \in D$. Then, there exists a nontrivial solution $h(\cdot) \in C^{2}\left(B\left(x_{0}, \rho\right) \subseteq D\right)$ satisfying $(55)$, for any $x \in \operatorname{Int} B\left(x_{0}, \rho\right)$. It relies on Lemma 1, noticing that (55) can be rewritten as

$$
(\vec{X})^{2}(h)(x)=a_{1}(x) h(x), x \in B\left(x_{0}, \rho\right) \subseteq D, a_{1} \in C^{1}(D),
$$

where $\vec{X}(h)(x):=\left\langle\partial_{x} h(x), X(x)\right\rangle$. It shows that any $a_{1}(\cdot)=\mu \in R$ is an eigenvalue for the linear differential operator $(\vec{X})^{2}$ acting on the space $C^{2}\left(B\left(x_{0}, \rho\right) \subseteq D\right)$. In particular, for $a_{1}(\cdot)=0 \in R$ and $X\left(x_{0}\right) \neq 0_{R^{n}}$, there exists a nontrivial solution $\left(\vec{X}(h) \neq 0, h \in C^{2}\left(B\left(x_{0}, \rho\right) \subseteq D\right)\right.$ ) fulfilling a second order PDE

$$
(\vec{X})^{2}(h)(x)=0, x \in B\left(x_{0}, \rho\right) \subseteq D .
$$

For the case $n \geq 2$, a nontrivial solution of (57) can be found such that $\vec{X}(h)(x) \neq$ const., for $x \in B\left(x_{0}, \rho\right) \subseteq$ $D$. It comes from the existence of a nontrivial first integral associated with ODE

$$
\frac{d G}{d \sigma}(\sigma, \lambda)=X(G(\sigma, \lambda)), G(0, \lambda)=\lambda \in B(0, \rho) \subseteq D, \sigma \in[-a, a] .
$$

\section{Conclusions and Further Developments}

In this paper, we have investigated the kernel of a polynomial differential equation involving a derivation in $R^{n}$ by solving the Cauchy Problem for the corresponding first order system of PDEs. Moreover, we have proved that the kernel representation has a special significance on the space of solutions to corresponding system of PDEs.

The mathematical framework developed in this work can be easily extended for the study of some higher-order hyperbolic, parabolic or Hamilton-Jacobi equations involving a finite set of derivations.

Funding: The APC was funded by University Politehnica of Bucharest, "PubArt" program.

Acknowledgments: The author would like to thank the referees for their precise remarks, which improved the presentation of this paper.

Conflicts of Interest: The author declares no conflict of interest.

\section{References}

1. Vârsan, C. Applications of Lie Algebras to Hyperbolic and Stochastic Differential Equations; Kluwer Academic Publishers: Amsterdam, The Netherlands, 1999.

2. Ijacu, D.; Vârsan, C. Smooth Mappings and Non $F_{T}$-Adapted Solutions Associated with Hamilton-Iacobi Stochastic Equations. In Proceedings of the IFIP TC7/WG7.2 International Working Conference on Analysis and Optimization of Differential Systems, Constanta, Romania, 10-14 September 2002; pp. 211-218.

3. Iftimie, B.; Marinescu, M.; Vârsan, C. Functionals Associated with Gradient Stochastic Flows and Nonlinear SPDEs. In Advanced Mathematical Methods for Finance; Di Nunno, G., Øksendal, B., Eds.; Springer: Berlin/Heidelberg, Germany, 2011; pp 397-415.

4. Parveen, S.; Akram, M.S. Linear subspaces of smooth vector fields as a kernel of some linear first order partial differential equations. Syst. Control Lett. 2012, 61, 86-91. [CrossRef]

5. Treanţă, S.; Vârsan, C. Weak small controls and approximations associated with controllable affine control systems. J. Differ. Equ. 2013, 255, 1867-1882. [CrossRef] 
6. Treanţă, S. Local uncontrollability for affine control systems with jumps. Int. J. Control 2017, 90, 1893-1902. [CrossRef]

7. Friedman, A. Stochastic Differential Equations and Applications; Academic Press: New York, NY, USA, 1975; Voulme 1.

8. Sussmann, H.J. Lie brackets and local controllability: A sufficient condition for scalar-input systems. SIAM J. Control Optim. 1983, 21, 686-713. [CrossRef]

9. Crandall, M.G.; Souganidis, P.E. Developments in the Theory of Nonlinear First-Order Partial Differential Equations; Differential Equations; North-Holland Math. Studies, 92; North-Holland: Amsterdam, The Netherlands, 1984; pp. 131-142.

10. Sontag, E.D. Mathematical Control Theory: Deterministic Finite Dimensional Systems; Texts Appl. Math. 6; Springer: New York, NY, USA, 1990.

11. Bressan, A.; Shen, W. On discontinuous differential equations. In Differential Inclusions and Optimal Control; Andres, J., Gorniewicz, L., Nistri, P., Eds.; Lecture Notes in Nonlinear Analysis, 2; N. Copernicus Univ.: Torun, Poland, 1998; pp. 73-87.

12. Nonlaopon, K. On the Inverse Ultrahyperbolic Klein-Gordon Kernel. Mathematics 2019, 7, 534. [CrossRef]

13. Xiang, S.; Liu, G. Numerical Solution of the Cauchy-Type Singular Integral Equation with a Highly Oscillatory Kernel Function. Mathematics 2019, 7,872.

14. Treanţă, S. Hamilton-Jacobi system of PDEs governed by higher-order Lagrangians. Transnatl. J. Math. Anal. Appl. 2017, 5, 1-15.

15. Treanţă, S. Higher-order Hamilton dynamics and Hamilton-Jacobi divergence PDE. Comput. Math. Appl. 2018, 75, 547-560. [CrossRef]

16. Treanţă, S. Noether-type first integrals associated with autonomous second-order Lagrangians. Symmetry 2019, 11, 1088. [CrossRef]

17. Treanţă, S. On the Kernel of a Polynomial of Scalar Derivations. Mathematics 2020, 8, 515. [CrossRef]

(C) 2020 by the author. Licensee MDPI, Basel, Switzerland. This article is an open access article distributed under the terms and conditions of the Creative Commons Attribution (CC BY) license (http:/ / creativecommons.org/licenses/by/4.0/). 